\title{
Some Recent Developments in Ambit Stochastics
}

\author{
Ole E. Barndorff-Nielsen, Emil Hedevang, Jürgen Schmiegel \\ and Benedykt Szozda
}

\begin{abstract}
Some of the recent developments in the rapidly expanding field of Ambit Stochastics are here reviewed. After a brief recall of the framework of Ambit Stochastics, two topics are considered: (i) Methods of modelling and inference for volatility/intermittency processes and fields; (ii) Universal laws in turbulence and finance in relation to temporal processes. This review complements two other recent expositions.
\end{abstract}

Keywords Ambit stochastics - Stochastic volatility/intermittency - Universality • Finance $\cdot$ Turbulence $\cdot$ Extended subordination $\cdot$ Metatimes $\cdot$ Time-change

\section{Introduction}

Ambit Stochastics is a general framework for the modelling and study of dynamic processes in space-time. The present paper outlines some of the recent developments in the area, with particular reference to finance and the statistical theory of turbulence. Two recent papers $[8,36]$ provide surveys that focus on other sides of Ambit Stochastics.

O.E. Barndorff-Nielsen $(\bowtie) \cdot$ E. Hedevang $\cdot$ B. Szozda

Department of Mathematics, Aarhus University, Ny Munkegade 118, 8000 Aarhus C, Denmark

e-mail: oebn@math.au.dk

E. Hedevang

e-mail: hedevang@math.au.dk

B. Szozda

e-mail: szozda@math.au.dk

J. Schmiegel

Department of Engineering, Aarhus University, Inge Lehmanns Gade 10,

8000 Aarhus C, Denmark

e-mail: schmiegl@eng.au.dk 
A key characteristic of the Ambit Stochastics framework, which distinguishes this from other approaches, is that beyond the most basic kind of random input it also specifically incorporates additional, often drastically changing, inputs referred to as volatility or intermittency.

Such "additional" random fluctuations generally vary, in time and/or in space, in regard to intensity (activity rate and duration) and amplitude. Typically the volatility/intermittency may be further classified into continuous and discrete (i.e. jumps) elements, and long and short term effects. In turbulence the key concept of energy dissipation is subsumed under that of volatility/intermittency.

The concept of (stochastic) volatility/intermittency is of major importance in many fields of science. Thus volatility/intermittency has a central role in mathematical finance and financial econometrics, in turbulence, in rain and cloud studies and other aspects of environmental science, in relation to nanoscale emitters, magnetohydrodynamics, and to liquid mixtures of chemicals, and last but not least in the physics of fusion plasmas.

As described here, volatility/intermittency is a relative concept, and its meaning depends on the particular setting under investigation. Once that meaning is clarified the question is how to assess the volatility/intermittency empirically and then to describe it in stochastic terms, for incorporation in a suitable probabilistic model. Important issues concern the modelling of propagating stochastic volatility/intermittency fields and the question of predictability of volatility/intermittency.

Section 2 briefly recalls some main aspects of Ambit Stochastics that are of relevance for the dicussions in the subsequent sections, and Sect. 3 illustrates some of the concepts involved by two examples. The modelling of volatility/intermittency and energy dissipation is a main theme in Ambit Stochastics and several approaches to this are discussed in Sect.4. A leading principle in the development of Ambit Stochastics has been to take the cue from recognised stylised features-or universality traits-in various scientific areas, particularly turbulence, as the basis for model building; and in turn to seek new such traits using the models as tools. We discuss certain universal features observed in finance and turbulence and indicate ways to reproduce them in Sect. 5. Section 6 concludes and provides an outlook.

\section{Ambit Stochastics}

\subsection{General Framework}

In terms of mathematical formulae, in its original form [17] (cf. also [16]) an ambit field is specified by

$$
Y(x, t)=\mu+\int_{A(x, t)} g(x, \xi, t, s) \sigma(\xi, s) L(d \xi d s)+Q(x, t)
$$




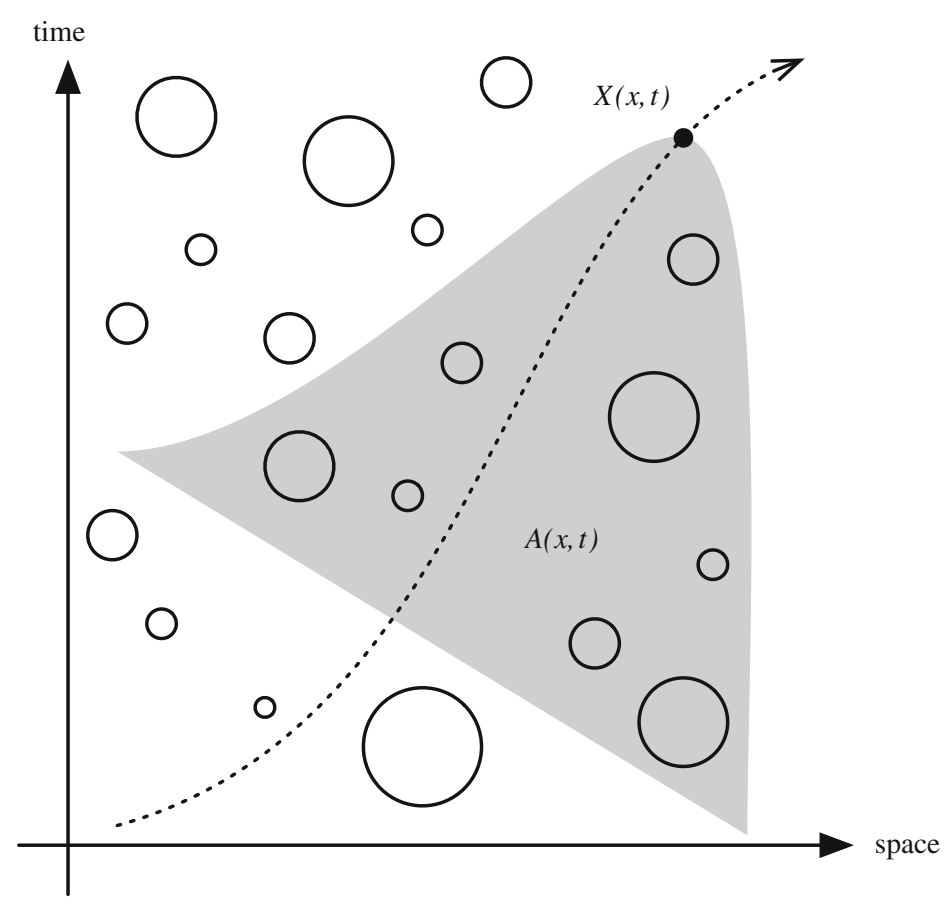

Fig. 1 A spatio-temporal ambit field. The value $Y(x, t)$ of the field at the point marked by the black $d o t$ is defined through an integral over the corresponding ambit set $A(x, t)$ marked by the shaded region. The circles of varying sizes indicate the stochastic volatility/intermittency. By considering the field along the dotted path in space-time an ambit process is obtained

where

$$
Q(x, t)=\int_{D(x, t)} q(x, \xi, t, s) \chi(\xi, s) d \xi d s .
$$

Here $t$ denotes time while $x$ gives the position in $d$-dimensional Euclidean space. Further, $A(x, t)$ and $D(x, t)$ are subsets of $\mathbb{R}^{d} \times \mathbb{R}$ and are termed ambit sets, $g$ and $q$ are deterministic weight functions, and $L$ denotes a Lévy basis (i.e. an independently scattered and infinitely divisible random measure). Further, $\sigma$ and $\chi$ are stochastic fields representing aspects of the volatility/intermittency. In Ambit Stochastics the models of the volatility/intermittency fields $\sigma$ and $\chi$ are usually themselves specified as ambit fields. We shall refer to $\sigma$ as the amplitude volatility component. Figure 1 shows a sketch of the concepts.

The development of $Y$ along a curve in space-time is termed an ambit process. As will be exemplified below, ambit processes are not in general semimartingales, even in the purely temporal case, i.e. where there is no spatial component $x$. 
In a recent extension the structure (1) is generalised to

$$
Y(x, t)=\mu+\int_{A(x, t)} g(x, \xi, t, s) \sigma(\xi, s) L_{T}(d \xi d s)+Q(x, t)
$$

where $Q$ is like (2) or the exponential thereof, and where $T$ is a metatime expressing a further volatility/intermittency trait. The relatively new concept of metatime is instrumental in generalising subordination of stochastic processes by time change (as discussed for instance in [22]) to subordination of random measures by random measures. We return to this concept and its applications in the next section and refer also to the discussion given in [8].

Note however that in addition to modelling volatility/intermittency through the components $\sigma, \chi$ and $T$, in some cases this may be supplemented by probability mixing or Lévy mixing as discussed in [12].

It might be thought that ambit sets have no role in purely temporal modelling. However, examples of their use in such contexts will be discussed in Sect. 3.

In many cases it is possible to choose specifications of the volatility/intermittency elements $\sigma, \chi$ and $T$ such that these are infinitely divisible or even selfdecomposable, making the models especially tractable analytically. We recall that the importance of the concept of selfdecomposability rests primarily on the possibility to represent selfdecomposable variates as stochastic integrals with respect to Lévy processes, see [32].

So far, the main applications of ambit stochastics has been to turbulence and, to a lesser degree, to financial econometrics and to bioimaging. An important potential area of applications is to particle transport in fluids.

\subsection{Existence of Ambit Fields}

The paper [25] develops a general theory for integrals

$$
X(x, t)=\int_{\mathbb{R}^{d} \times \mathbb{R}} h(x, y, t, s) M(d x d x)
$$

where $h$ is a predictable stochastic function and $M$ is a dispersive signed random measure. Central to this is that the authors establish a notion of characteristic triplet of $M$, extending that known in the purely temporal case. A major problem solved in that regard has been to merge the time and space aspects in a general and tractable fashion. Armed with that notion they determine the conditions for existence of the integral, analogous to those in [37] but considerably more complicated to derive and apply. An important property here is that now predictable integrands are allowed (in the purely temporal case this was done in [23]). Applications of the theory to Ambit Stochastics generally, and in particular to superposition of stochastic volatility models, is discussed. 
Below we briefly discuss how the metatime change is incorporated in the framework of [25]. Suppose that $L=\left\{L(A) \mid A \in \mathscr{B}_{b}\left(\mathbb{R}^{d+1}\right\}\right.$ is a real-valued, homogeneous Lévy basis with associated infinitely divisible law $\mu \in I D\left(\mathbb{R}^{d+1}\right)$, that is $L\left([0,1]^{d+1}\right)$ is equal in law to $\mu$. Let $(\gamma, \Sigma, v)$ be the characteristic triplet of $\mu$. Thus $\gamma \in \mathbb{R}, \Sigma \geq 0$ and $v$ is a Lévy measure on $\mathbb{R}$.

Suppose that $\mathbf{T}=\left\{\mathbf{T}(A) \mid A \in \mathscr{B}\left(\mathbb{R}^{d+1}\right\}\right.$ is a random meta-time associated with a homogeneous, real-valued, non-negative Lévy basis $T=\{T(A) \mid A \in$ $\left.\mathscr{B}\left(\mathbb{R}^{d+1}\right)\right\}$. That is the sets $\mathbf{T}(A)$ and $\mathbf{T}(B)$ are disjoint whenever $A, B \in \mathscr{B}\left(\mathbb{R}^{d+1}\right)$ are disjoint, $\mathbf{T}\left(\cup_{n=0}^{\infty} A_{n}\right)=\cup_{n=0}^{\infty} \mathbf{T}\left(A_{n}\right)$ whenever $A_{n}, \cup_{n=0}^{\infty} A_{n} \in \mathscr{B}\left(\mathbb{R}^{d+1}\right)$ and $T(A)=\operatorname{Leb}_{d+1}(\mathbf{T}(A))$ for all $A \in \mathscr{B}\left(\mathbb{R}^{d+1}\right)$. Here and in what follows, Leb $k$ denotes the Lebesgue measure on $\mathbb{R}^{k}$. For the details on construction of random meta-times cf. [11]. Suppose also that $\lambda \in I D(\mathbb{R})$ is the law associated to $T$ and that $\lambda \sim I D(\beta, 0, \rho)$. Thus $\beta \geq 0$ and $\rho$ is a Lévy measure such that $\rho\left(\mathbb{R}_{-}\right)=0$ and $\int_{\mathbb{R}}(1 \wedge x) \rho(d x)<\infty$.

Now, by $\left[11\right.$, Theorem 5.1] we have that $L_{T}=\left\{L(\mathbf{T}(A)) \mid A \in \mathscr{B}\left(\mathbb{R}^{d+1}\right)\right\}$ is a homogeneous Lévy basis associated to $\mu^{\#}$ with $\mu^{\#} \sim I D\left(\gamma^{\#}, \Sigma^{\#}, v^{\#}\right)$ and characteristics given by

$$
\begin{aligned}
\gamma^{\#} & =\beta \gamma+\int_{0}^{\infty} \int_{|x| \leq 1} x \mu^{s}(d x) \rho(d s) \\
\Sigma^{\#} & =\beta \Sigma \\
\nu^{\#}(B) & =\beta v(B)+\int_{0}^{\infty} \mu^{s}(B) \rho(d s), \quad B \in \mathscr{B}\left(\mathbb{R}^{d+1} \backslash\{0\}\right),
\end{aligned}
$$

where $\mu^{s}$ is given by $\widehat{\mu^{s}}=\widehat{\mu}^{s}$ for any $s \geq 0$.

Finally, suppose that $\sigma(x, t)$ is predictable and that $L_{T}$ has no fixed times of discontinuity (see [25]). By rewriting the stochastic integral in the right-hand side of (3) as

$$
X(x, t)=\int_{\mathbb{R}^{d+1}} H(x, \xi, t, s) L_{T}(d \xi d s),
$$

with $H(x, \xi, t, s)=\mathbf{1}_{A(x, t)}(\xi, s) g(x, \xi, t, s) \sigma(\xi, s)$ we can use [25, Theorem 4.1]. Observe that the assumption that $\sigma$ is predictable is enough as both $A(x, t)$ and $g(x, \xi, t, s)$ are deterministic. This gives us that $X$ is well defined for all $(x, t)$ if the following hold almost surely for all $(x, t) \in \mathbb{R}^{d+1}$ :

$$
\begin{gathered}
\int_{\mathbb{R}^{d+1}}\left|H(x, \xi, t, s) \gamma^{\#}+\int_{\mathbb{R}}[\tau(H(x, \xi, t, s) y)-H(x, \xi, t, s) \tau(y)] v^{\#}(d y)\right| d \xi d s<\infty \\
\int_{\mathbb{R}^{d+1}} H^{2}(x, \xi, t, s) \Sigma^{\#} d \xi d s<\infty \\
\int_{\mathbb{R}^{d+1}} \int_{\mathbb{R}^{2}}\left(1 \wedge(H(x, \xi, t, s) y)^{2} \nu^{\#}(d y) d \xi d s<\infty\right.
\end{gathered}
$$




\section{Illustrative Examples}

We can briefly indicate the character of some of the points on Ambit Stochastics made above by considering the following simple model classes.

\subsection{BSS and LSS Processes}

Stationary processes of the form

$$
Y(t)=\int_{-\infty}^{t} g(t-s) \sigma(s) B_{T}(d s)+\int_{-\infty}^{t} q(t-s) \sigma(s)^{2} d s .
$$

are termed Brownian semistationary processes-or BSS for short. Here the setting is purely temporal and $B_{T}$ is the time change of Brownian motion $B$ by a chronometer $T$ (that is, an increasing, càdlàg and stochastically continuous process ranging from $-\infty$ to $\infty$ ), and the volatility/intermittency process $\sigma$ is assumed stationary. The components $\sigma$ and $T$ represent respectively the amplitude and the intensity of the volatility/intermittency. If $T$ has stationary increments then the process $Y$ is stationary. The process (7) can be seen as a stationary analogue of the BNS model introduced by Barndorff-Nielsen and Shephard [14].

Note that in case $T$ increases by jumps only, the infinitesimal of the process $B_{T}$ cannot be reexpressed in the form $\chi(s) B(d s)$, as would be the case if $T$ was of type $T_{t}=\int_{0}^{t} \psi(u) d u$ with $\chi=\sqrt{\psi}$.

Further, for the exemplification we take $g$ to be of the gamma type

$$
g(s)=\frac{\lambda^{\nu}}{\Gamma(\nu)} s^{\nu-1} e^{-\lambda s} 1_{(0, \infty)}(s) .
$$

Subject to a weak (analogous to (4)) condition on $\sigma$, the stochastic integral in (7) will exist if and only if $v>1 / 2$ and then $Y$ constitutes a stationary process in time. Moreover, $Y$ is a semimartingale if and only if $v$ does not lie in one of the intervals $(1 / 2,1)$ and $(1,3 / 2]$. Note also that the sample path behaviour is drastically different between the two intervals, since, as $t \rightarrow 0, g(t)$ tends to $\infty$ when $v \in(1 / 2,1)$ and to 0 when $v \in(1,3 / 2]$. Further, the sample paths are purely discontinuous if $v \in(1 / 2,1)$ but purely continuous (of Hölder index $H=v-1 / 2)$ when $v \in(1,3 / 2)$.

The cases where $v \in(1 / 2,1)$ have a particular bearing in the context of turbulence, the value $v=5 / 6$ having a special role in relation to the Kolmogorov-Obukhov theory of statistical turbulence, cf. [3, 33].

The class of processes obtained by substituting the Brownian motion in (7) by a Lévy process is referred to as the class of Lévy semistationary processes-or LSS processes for short. Such processes are discussed in [8, 24, Sect. 3.7] and references therein. 


\subsection{Trawl Processes}

The simplest non-trivial kind of ambit field is perhaps the trawl process, introduced in [2]. In a trawl process, the kernel function and the volatility field are constant and equal to 1 , and so the process is given entirely by the ambit set and the Lévy basis. Specifically, suppose that $L$ is a homogeneous Lévy basis on $\mathbb{R}^{d} \times \mathbb{R}$ and that $A \subseteq \mathbb{R}^{d} \times \mathbb{R}$ is a Borel subset with finite Lebesgue measure, then we obtain a trawl process $Y$ by letting $A(t)=A+(0, t)$ and

$$
Y(t)=\int_{A(t)} L(d \xi d s)=\int 1_{A}(\xi, t-s) L(d \xi d s)=L(A(t))
$$

The process is by construction stationary. Depending on the purpose of the modelling, the time component of the ambit set $A$ may or may not be supported on the negative real axis. When the time component of $A$ is supported on the negative real axis, we obtain a causal model. Despite their apparent simplicity, trawl processes possess enough flexibility to be of use. If $L^{\prime}$ denotes the seed ${ }^{1}$ of $L$, then the cumulant function (i.e. the distinguished logarithm of the characteristic function) of $Y$ is given by

$$
C\{\zeta \neq Y(t)\}=|A| C\left\{\zeta \neq L^{\prime}\right\} .
$$

Here and later, $|A|$ denotes the Lebesgue measure of the set $A$. For the mean, variance, autocovariance and autocorrelation it follows that

$$
\begin{aligned}
\mathbb{E}[Y(t)] & =|A| \mathbb{E}\left[L^{\prime}\right], \\
\operatorname{var}(Y(t)) & =|A| \operatorname{var}\left(L^{\prime}\right), \\
r(t) & :=\operatorname{cov}(Y(t), Y(0))=|A \cap A(t)| \operatorname{var}\left(L^{\prime}\right), \\
\rho(t) & :=\frac{\operatorname{cov}(Y(t), Y(0))}{\operatorname{var}(Y(0))}=\frac{|A \cap A(t)|}{|A|} .
\end{aligned}
$$

From this we conclude the following. The one-dimensional marginal distribution is determined entirely in terms of the size (not shape) of the ambit set and the distribution of the Lévy seed; given any infinitely divisible distribution there exists trawl processes having this distribution as the one-dimensional marginal; and the autocorrelation is determined entirely by the size of the overlap of the ambit sets, that is, by the shape of the ambit set $A$. Thus we can specify the autocorrelation and marginal distribution independently of each other. It is, for example, easy to construct a trawl process with the same autocorrelation as the OU process, see [2, 8] for more results and details. By using integer-valued Lévy bases, integer-valued

\footnotetext{
${ }^{1}$ The Lévy seed $L^{\prime}(x)$ at $x$ of a Lévy basis $L$ with control measure $v$ is a random variable with the property that $C\{\zeta \neq L(A)\}=\int_{A} C\left\{\zeta \neq L^{\prime}(x)\right\} \nu(d x)$. For a homogeneous Lévy basis, the distribution of the seed is independent of $x$.
} 
trawl processes are obtained. These processes are studied in detail in [5] and applied to high frequency stock market data.

We remark, that $Y(x, t)=L(A+(x, t))$ is an immediate generalisation of trawl processes to trawl fields. It has the same simple properties as the trawl process.

Trawl processes can be used to directly model an object of interest, for example, the exponential of the trawl process has been used to model the energy dissipation, see the next section, or they can be used as a component in a composite model, for example to model the volatility/intermittency in a Brownian semistationary process.

\section{Modelling of Volatility/Intermittency/Energy Dissipation}

A very general approach to specifying volatility/intermittency fields for inclusion in an ambit field, as in (1), is to take $\tau=\sigma^{2}$ as being given by a Lévy-driven Volterra field, either directly as

$$
\tau(x, t)=\int_{\mathbb{R}^{2} \times \mathbb{R}} f(x, \xi, t, s) L(d \xi, d s)
$$

with $f$ positive and $L$ a Lévy basis (different from $L$ in (1), or in exponentiated form

$$
\tau(x, t)=\exp \left(\int_{\mathbb{R}^{d} \times \mathbb{R}} f(x, \xi, t, s) L(d \xi, d s)\right) .
$$

When the goal is to have stationary volatility/intermittency fields, such as in modelling homogeneous turbulence, that can be achieved by choosing $L$ to be homogeneous and $f$ of translation type. However, the potential in the specifications (12) and (13) is much wider, giving ample scope for modelling inhomogeneous fields, which are by far the most common, particularly in turbulence studies. Inhomogeneity can be expressed both by not having $f$ of translation type and by taking the Lévy basis $L$ inhomogeneous.

In the following we discuss two aspects of the volatility/intermittency modelling issue. Trawl processes have proved to be a useful tool for the modelling of volatility/intermittency and in particular for the modelling of the energy dissipation, as outlined in Sect.4.1. Section 4.2 reports on a recent paper on relative volatility/intermittency. In Sect. 4.3 we discuss the applicability of selfdecomposability to the construction of volatility/intermittency fields. 


\subsection{The Energy Dissipation}

In [31] it has been shown that exponentials of trawl processes are able to reproduce the main stylized features of the (surrogate) energy dissipation observed for a wide range of datasets. Those stylized features include the one-dimensional marginal distributions and the scaling and self-scaling of the correlators.

The correlator of order $(p, q)$ is defined by

$$
c_{p, q}(s)=\frac{\mathbb{E}\left[\varepsilon(t)^{p} \varepsilon(t+s)^{q}\right]}{\mathbb{E}\left[\varepsilon(t)^{p}\right] \mathbb{E}\left[\varepsilon(t+s)^{q}\right]} .
$$

The correlator is a natural analogue to the autocorrelation when one considers a purely positive process. In turbulence it is known (see the reference cited in [31]) that the correlator of the surrogate energy dissipation displays a scaling behaviour for a certain range of lags,

$$
c_{p, q}(s) \propto s^{-\tau(p, q)}, \quad T_{\text {small }} \ll s \ll T_{\text {large }},
$$

where $\tau(p, q)$ is the scaling exponent. The exponent $\tau(1,1)$ is the so-called intermittency exponent. Typical values are in the range 0.1 to 0.2 . The intermittency exponent quantifies the deviation from Kolmogorov's 1941 theory and emphasizes the role of intermittency (i.e. volatility) in turbulence. In some cases, however, the scaling range of the correlators can be quite small and therefore it can be difficult to determine the value of the scaling exponents, especially when $p$ and $q$ are large. Therefore one also considers the correlator of one order as a function of a correlator of another order. In this case, self-scaling is observed, i.e., the one correlator is proportional to a power of the other correlator,

$$
c_{p, q}(s) \propto c_{p^{\prime}, q^{\prime}}(s)^{\tau\left(p, q ; p^{\prime}, q^{\prime}\right)}
$$

where $\tau\left(p, q ; p^{\prime}, q^{\prime}\right)$ is the self-scaling exponent. The self-scaling exponents have turned out to be much easier to determine from data than the scaling exponents, and like the scaling exponents, the self-scaling exponents have proved to be key fingerprints of turbulence. They are essentially universal in that they vary very little from one dataset to another, covering a large range of the so-called Reynolds numbers, a dimensionless quantity describing the character of the flow.

In [31] the surrogate energy dissipation $\varepsilon$ is, more specifically, modelled as

$$
\varepsilon(t)=\exp (L(A(t)))
$$

where $L$ is a homogeneous Lévy basis on $\mathbb{R} \times \mathbb{R}$ and $A(t)=A+(0, t)$ for a bounded set $A \subset \mathbb{R} \times \mathbb{R}$. The ambit set $A$ is given as

$$
A=\left\{(x, t) \in \mathbb{R} \times \mathbb{R} \mid 0 \leq t \leq T_{\text {large }},-f(t) \leq x \leq f(t)\right\}
$$




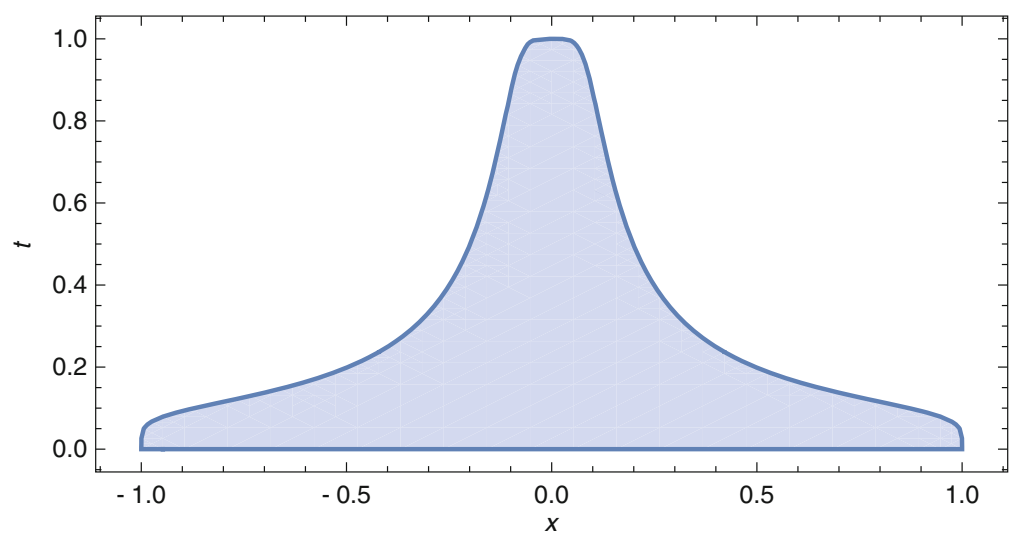

Fig. 2 The shaded region marks the ambit set $A$ from (18) defined by (19) where the parameters are chosen to be $T_{\text {large }}=1, T_{\text {small }}=0.1$ and $\theta=5$

where $T_{\text {large }}>0$. For $T_{\text {large }}>T_{\text {small }}>0$ and $\theta>0$, the function $f$ is defined as

$$
f(t)=\left(\frac{1-\left(t / T_{\text {large }}\right)^{\theta}}{1+\left(t / T_{\text {small }}\right)^{\theta}}\right)^{1 / \theta}, \quad 0 \leq t \leq T_{\text {large }} .
$$

The shape of the ambit set is chosen so that the scaling behaviour (15) of the correlators is reproduced. The exact values of the scaling exponents are determined from the distribution of the Lévy seed of the Lévy basis. The two parameters $T_{\text {small }}$ and $T_{\text {large }}$ determine the size of the small and large scales of turbulence: in between we have the inertial range. The final parameter $\theta$ is a tuning parameter which accounts for the lack of perfect scaling and essentially just allows for a better fit. (Perfect scaling is obtained in the limit $\theta \rightarrow \infty$ ). See Fig. 2 for an example. Furthermore, self-scaling exponents are predicted from the shape, not location and scale, of the one-point distribution of the energy dissipation alone.

To determine a proper distribution of the Lévy seed of $L$, it is in [31] shown that the one-dimensional marginal of the logarithm of the energy dissipation is well described by a normal inverse Gaussian distribution, i.e. $\log \varepsilon(t) \sim \operatorname{NIG}(\alpha, \beta, \mu, \delta)$, where the shape parameters $\alpha$ and $\beta$ are the same for all datasets (independent of the Reynolds number). Thus the shape of the distribution of the energy dissipation is a newly discovered universal feature of turbulence. Thus we see that $L$ should be a normal inverse Gaussian Lévy basis whose parameters are given by the observed distribution of $\log \varepsilon(t)$. This completely specifies the parameters of (17). 


\subsection{Realised Relative Volatility/Intermittency/Energy Dissipation}

By its very nature, volatility/intermittency is a relative concept, delineating variation that is relative to a conceived, simpler model. But also in a model for volatility/intermittency in itself it is relevant to have the relative character in mind, as will be further discussed below. We refer to this latter aspect as relative volatility/intermittency and will consider assessment of that by realised relative volatility/intermittency which is defined in terms of quadratic variation. The ultimate purpose of the concept of relative volatility/intermittency is to assess the volatility/intermittency or energy dissipation in arbitrary subregions of a region $C$ of spacetime relative to the total volatility/intermittency/energy dissipation in $C$. In the purely temporal setting the realised relative volatility/intermittency is defined by

$$
\left[Y_{\delta}\right]_{t} /\left[Y_{\delta}\right]_{T}
$$

where $\left[Y_{\delta}\right]_{t}$ denotes the realised quadratic variation of the process $Y$ observed with lag $\delta$ over a time interval $[0, t]$. We refer to this quantity as RRQV (for realised relative quadratic volatility).

As mentioned in Example 1, in case $g$ is the gamma kernel (8) with $v \in(1 / 2,1) \cup$ $(1,3 / 2]$ then the BSS process (7) is not a semimartingale. In particular, if $v \in$ $(1 / 2,1)$ - the case of most interest for the study of turbulence-the realised quadratic variation $\left[Y_{\delta}\right]_{t}$ does not converge as it would if $Y$ was a semimartingale; in fact it diverges to infinity whereas in the semimartingale case it will generally converge to the accumulated volatility/intermittency

$$
\sigma_{t}^{2+}=\int_{0}^{t} \sigma_{s}^{2} d s,
$$

which is an object of key interest (in turbulence it represents the coarse-grained energy dissipation). However the situation can be remedied by adjusting $\left[Y_{\delta}\right]_{t}$ by a factor depending on $v$; in wide generality it holds that

$$
c \delta^{2(1-v)}\left[Y_{\delta}\right]_{t} \stackrel{p}{\longrightarrow} \sigma_{t}^{2+}
$$

as $\delta \longrightarrow 0$, with $x=\lambda^{-1} 2^{2(v-1 / 2)}(\Gamma(v)+\Gamma(v+1 / 2)) / \Gamma(2 v-1) \Gamma(3 / 2-1)$. To apply this requires knowledge of the value of $v$ and in general $v$ must be estimated with sufficient precision to ensure that substituting the estimate for the theoretical value of $v$ in (22) will still yield convergence in probability. Under relatively mild conditions that is possible, as discussed in [26] and the references therein. An important aspect of formula (20) is that its use does not involve knowledge of $v$ as the adjustment factor cancels out (Fig. 3).

Convergence in probability and a central limit theorem for the RRQV is established in [10]. Figure 2 illustrates its use, for two sections of the "Brookhaven" dataset, 

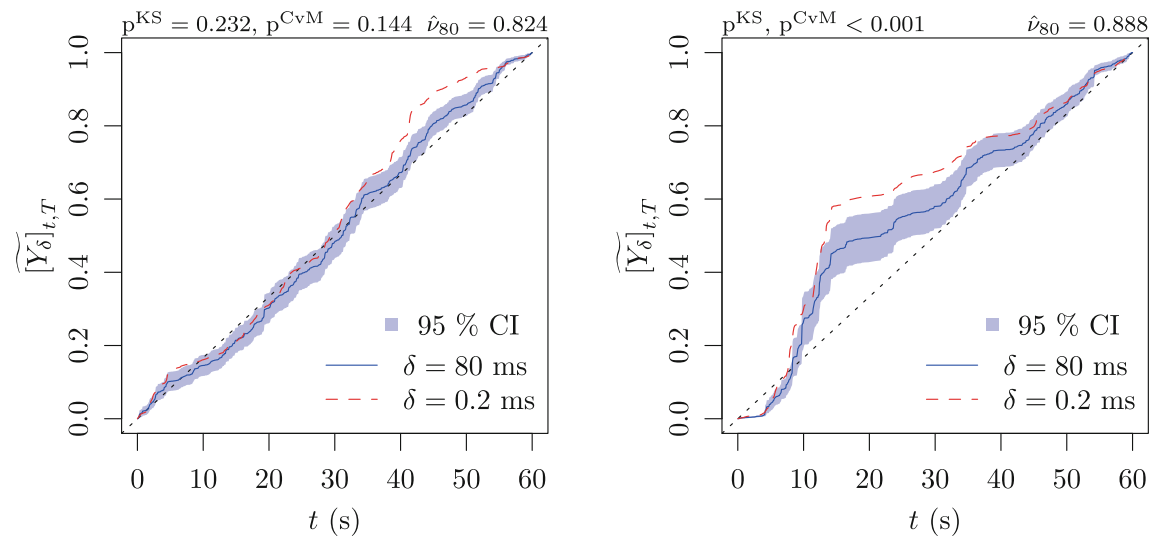

Fig. 3 Brookhaven turbulence data periods 18 and 25-RRQV and $95 \%$ confidence intervals

one where the volatility effect was deemed by eye to be very small and one where it appeared strong. (The "Brookhaven" dataset consist of 20 million one-point measurements of the longitudinal component of the wind velocity in the atmospheric boundary layer, $35 \mathrm{~m}$ above ground. The measurements were performed using a hot-wire anemometer and sampled at $5 \mathrm{kHz}$. The time series can be assumed to be stationary. We refer to [27] for further details on the dataset; the dataset is called no. 3 therein).

\subsection{Role of Selfdecomposability}

If $\tau$ is given by (12) it is automatically infinitely divisible, and selfdecomposable provided $L$ has that property; whereas if $\tau$ is defined by (13) it will only in exceptional cases be infinitely divisible.

A non-trivial example of such an exceptional case is the following. The Gumbel distribution with density

$$
f(x)=\frac{1}{b} \exp \left(\frac{x-a}{b}-\exp \left(\frac{x-a}{b}\right)\right),
$$

where $a \in \mathbb{R}$ and $b>0$ is infinitely divisible [39]. In [31] it was demonstrated that the one-dimensional marginal distribution of the logarithm of the energy dissipation is accurately described by a normal inverse Gaussian distribution. One may also show (not done here) that the Gumbel distribution with $b=2$ provides another fit that is nearly as accurate as the normal inverse Gaussian. Furthermore, if $X$ is a Gumbel random variable with $b=2$, then $\exp (X)$ is distributed as the square of an exponential random variable, hence also infinitely divisible by [39]. Therefore, if 
the Lévy basis $L$ in (17) is chosen so that $L(A)$ follows a Gumbel distribution with $b=2$, then $\exp (L(A(t)))$ will be infinitely divisible.

For a general discussion of selfdecomposable fields we refer to [13]. See also [32] which provides a survey of when a selfdecomposable random variable can be represented as a stochastic integral, like in (12). Representations of that kind allow, in particular, the construction of field-valued processes of OU or supOU type that may be viewed as propagating, in time, an initial volatility/intermittency field defined on the spatial component of space-time for a fixed time, say $t=0$. Similarly, suppose that a model has been formulated for the time-wise development of a stochastic field at a single point in space. One may then seek to define a field on space-time such that at every other point of space the time-wise development of the field is stochastically the same as at the original space point and such that the field as a whole is stationary and selfdecomposable.

Example 1 (One dimensional turbulence) Let $Y$ denote an ambit field in the tempospatial case where the spatial dimension is 1 , and assume that for a preliminary purely temporal model $X$ of the same turbulent phenomenon a model has been formulated for the squared amplitude volatility component, say $\omega$. It may then be desirable to devise $Y$ such that the volatility/intermittency field $\tau=\sigma^{2}$ is stationary and infinitely divisible, and such that for every spatial position $x$ the law of $\tau(x, \cdot)$ is identical to that formulated for the temporal setting, i.e. $\omega$. If the temporal process is selfdecomposable then, subject to a further weak condition (see [13]), such a field can be constructed.

To sketch how this may proceed, recall first that the classical definition of selfdecomposability of a process $X$ says that all the finite-dimensional marginal distributions of $X$ should be selfdecomposable. Accordingly, due to a result by [38], for any finite set of time points $\hat{u}=\left(u_{1}, \ldots, u_{n}\right)$ the selfdecomposable vector variable $X(\hat{u})=\left(X\left(u_{1}\right), \ldots, X\left(u_{n}\right)\right)$ has a representation

$$
X(\hat{u})=\int_{0}^{\infty} e^{-\xi} L(d \xi, \hat{u})
$$

for some $n$-dimensional Lévy process $L(\cdot, \hat{u})$, provided only that the Lévy measure of $X(\hat{u})$ has finite log-moment. We now assume this to be the case and that $X$ is stationary

Next, for fixed $\hat{u}$, let $\{\tilde{L}(x, \hat{u}) \mid x \in \mathbb{R}\}$, be the $n$-dimensional Lévy process having the property that the law of $\tilde{L}(1, \hat{u})$ is equal to the law of $X(\hat{u})$. Then the integral

$$
X(x, \hat{u})=\int_{-\infty}^{x} e^{-\xi} \tilde{L}(d \xi, \hat{u})
$$

exists and the process $\{X(x, \hat{u}) \mid x \in \mathbb{R}\}$ will be stationary-of Ornstein-Uhlenbeck type-while for each $x$ the law of $X(x, \hat{u})$ will be the same as that of $X(\hat{u})$. 
However, off hand the Lévy processes $\tilde{L}(\cdot, \hat{u})$ corresponding to different sets $\hat{u}$ of time points may have no dynamic relationship to each other, while the aim is to obtain a stationary selfdecomposable field $X(x, t)$ such that $X(x, \cdot)$ has the same law as $X$ for all $x \in \mathbb{R}$. But, arguing along the lines of theorem 3.4 in [9], it is possible to choose the representative processes $\tilde{L}(\cdot, \hat{u})$ so that they are all defined on a single probability space and are consistent among themselves (in analogy to Kolmogorov's consistency result); and that establishes the existence of the desired field $X(x, t)$. Moreover, $X(\cdot, \cdot)$ is selfdecomposable, as is simple to verify.

The same result can be shown more directly using master Lévy measures and the associated Lévy-Ito representations, cf. [13].

Example 2 Assume that $X$ has the form

$$
X(u)=\int_{-\infty}^{u} g(u-\xi) L(d \xi)
$$

where $L$ is a Lévy process.

It has been shown in [13] that, in this case, provided $g$ is integrable with respect to the Lebesgue measure, as well as to $L$, and if the Fourier transform of $g$ is nonvanishing, then $X$, as a process, is selfdecomposable if and only if $L$ is selfdecomposable. When that holds we may, as above, construct a selfdecomposable field $X(x, t)$ with $X(x, \cdot) \sim X(\cdot)$ for every $x \in \mathbb{R}$ and $X(\cdot, t)$ of OU type for every $t \in \mathbb{R}$.

As an illustration, suppose that $g$ is the gamma kernel (8) with $v \in(1 / 2,1)$. Then the Fourier transform of $g$ is

$$
\hat{g}(\zeta)=(1-i \zeta / \lambda)^{-v}
$$

and hence, provided that $L$ is such that the integral (24) exists, the field $X(x, u)$ is stationary and selfdecomposable, and has the OU type character described above.

\section{Time Change and Universality in Turbulence and Finance}

\subsection{Distributional Collapse}

In [4], Barndorff-Nielsen et al. demonstrate two properties of the distributions of increments $\Delta_{\ell} X(t)=X(t)-X(t-\ell)$ of turbulent velocities. Firstly, the increment distributions are parsimonious, i.e., they are described well by a distribution with few parameters, even across distinct experiments. Specifically it is shown that the 
four-parameter family of normal inverse Gaussian distributions $(\mathrm{NIG}(\alpha, \beta, \mu, \delta))$ provides excellent fits across a wide range of lags $\ell$,

$$
\Delta_{\ell} X \sim \operatorname{NIG}(\alpha(\ell), \beta(\ell), \mu(\ell), \delta(\ell))
$$

Secondly, the increment distributions are universal, i.e., the distributions are the same for distinct experiments, if just the scale parameters agree,

$$
\Delta_{\ell_{1}} X_{1} \sim \Delta_{\ell_{2}} X_{2} \quad \text { if and only if } \quad \delta_{1}\left(\ell_{1}\right)=\delta_{2}\left(\ell_{2}\right),
$$

provided the original velocities (not increments) have been non-dimensionalized by standardizing to zero mean and unit variance. Motivated by this, the notion of stochastic equivalence class is introduced.

The line of study initiated in [4] is continued in [16], where the analysis is extended to many more data sets, and it is observed that

$$
\Delta_{\ell_{1}} X_{1} \sim \Delta_{\ell_{2}} X_{2} \quad \text { if and only if } \quad \operatorname{var}\left(\Delta_{\ell_{1}} X_{1}\right) \sim \operatorname{var}\left(\Delta_{\ell_{2}} X_{2}\right)
$$

which is a simpler statement than (26), since it does not involve any specific distribution. In [21], Barndorff-Nielsen et al. extend the analysis from fluid velocities in turbulence to currency and metal returns in finance and demonstrate that (27) holds when $X_{i}$ denotes the log-price, so increments are log-returns. Further corroboration of the existence of this phenomenon in finance is presented in the following subsection.

A conclusion from the cited works is that within the context of turbulence or finance there exists a family of distributions such that for many distinct experiments and a wide range of lags, the corresponding increments are distributed according to a member of this family. Moreover, this member is uniquely determined by the variance of the increments.

Up till recently these stylised features had not been given any theoretical background. However, in [20], a class of stochastic processes is introduced that exactly has the rescaling property in question.

\subsection{A First Look at Financial Data from SP500}

Motivated by the developments discussed in the previous subsection, in the following we complement the analyses in [4, 16, 21] with 29 assets from Standard \& Poor's 500 stock market index. The following assets were selected for study: AA, AIG, AXP, BA, BAC, C, CAT, CVX, DD, DIS, GE, GM, HD, IBM, INTC, JNJ, JPM, KO, MCD, MMM, MRK, MSFT, PG, SPY, T, UTX, VZ, WMT, XOM. For each asset, between 7 and 12 years of data is available. A sample time series of the log-price of asset $\mathrm{C}$ is displayed in Fig. 4, where the thin vertical line marks the day 2008-01-01. 


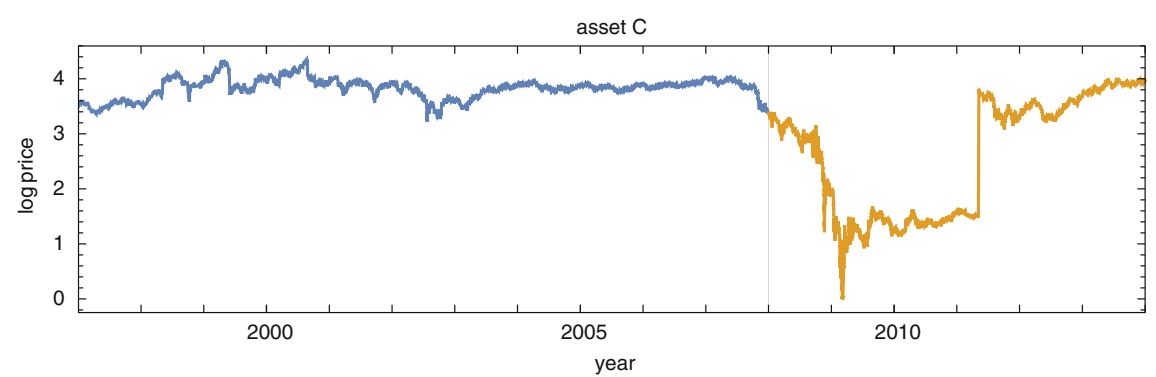

Fig. 4 Time series of the $\log$-price of asset $\mathrm{C}$ on an arbitrary scale. The thin vertical line marks the day 2008-01-01 and divides the dataset into the two subsets "pre" (blue) and "post" (yellow)

Asset $\mathrm{C}$ is found to be representative of the feature of all the other datasets. Each dataset is divided into two subsets: the "pre" subset consisting of data from before 2008-01-01 and the "post" subset consisting of data from after 2008-01-01. This subdivision was chosen since the volatility in the "post" dataset is visibly higher than in the "pre" dataset, presumably due to the financial crisis. The data has been provided by Lunde (Aarhus University), see also [29].

Figure 5 shows that the distributions of log-returns across a wide range of lags ranging from $1 \mathrm{~s}$ to approximately $4.5 \mathrm{~h}$ are quite accurately described by normal
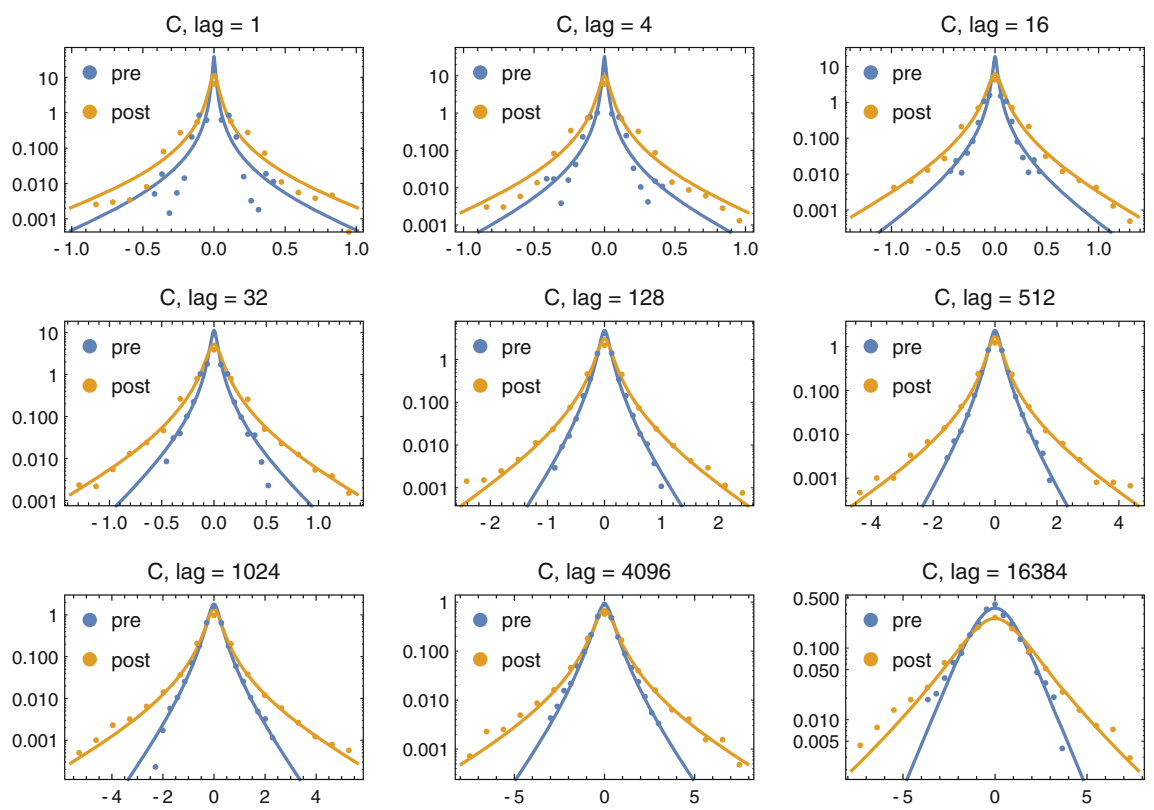

Fig. 5 Probability densities on a log-scale for the log-returns of asset $\mathrm{C}$ at various lags ranging from 1 to $16384 \mathrm{~s}$. The dots denote the data and the solid line denotes the fitted NIG distribution. Blue and yellow denote the "pre" and "post" datasets, respectively. The log-returns have been multiplied with 100 in order to un-clutter the labeling of the $x$-axes 
inverse Gaussian distributions, except at the smallest lags where the empirical distributions are irregular. We suspect this is due to market microstructure noise. The accuracy of the fits is not surprising given that numerous publications have demonstrated the applicability of the generalised hyperbolic distribution, in particular the subfamily consisting of the normal inverse Gaussian distributions, to describe financial datasets. See for example [1, 14, 15, 28, 40]. We note the transition from a highly peaked distribution towards the Gaussian as the lag increases.

Next, we see on Fig. 6 that the distributions at the same lag of the log-returns for the 29 assets are quite different, that is, they do not collapse onto the same curve. This holds for both the "pre" and the "post" datasets. However, the transition from a highly peaked distribution at small lags towards a Gaussian at large lags hints that a suitable change of time, though highly nonlinear, may cause such a collapse. Motivated by the observations in [21] we therefore consider the variance of the log-returns as a function of the lag. Figure 7 shows how the variance depends on the lag. Except at the smallest lags, a clear power law is observed. The behaviour at the smallest lags is due to market microstructure noise [29]. Nine variances have been selected to represent most of the variances observed in the 29 assets. For each selected variance and each asset the corresponding lag is computed. We note that for the smallest lags/variances this is not without difficulty since for some of the assets the slope approaches zero.

Finally, Fig. 8 displays the distributions of log-returns where the lag for each asset has been chosen such that the variance is the one specified in each subplot. The difference between Figs. 6 and 8 is pronounced. We see that for both the "pre" and the "post" dataset, the distributions corresponding to the same variance tend to be the same. Furthermore, when the "pre" and "post" datasets are displayed together, essentially overlaying the top part of Fig. 8 with the bottom part, a decent overlap is still observed. So while the distributions in Fig. 8 do not collapse perfectly onto the same curve for all the chosen variances, in contrast to what is the case for velocity increments in turbulence (see [4]), we are invariably led to the preliminary conclusion that also in the case of the analysed assets from S\&P500, a family of distributions exists such that all distributions of log-returns are members of this family and such that the variance of the log-returns uniquely determines this member. The lack of collapse at the smaller variances may in part be explained by the difficulty in reading off the corresponding lags.

The observed parsimony and in particular universality has implications for modelling since any proper model should possess both features. Within the context of turbulence, BSS-processes have been shown to be able to reproduce many key features of turbulence, see [35] and the following subsection for a recent example. The extent to which BSS-processes in general possess universality is still ongoing research [20] but results indicate that BSS-processes and in general LSS-process are good candidates for models where parsimony and universality are desired features. 

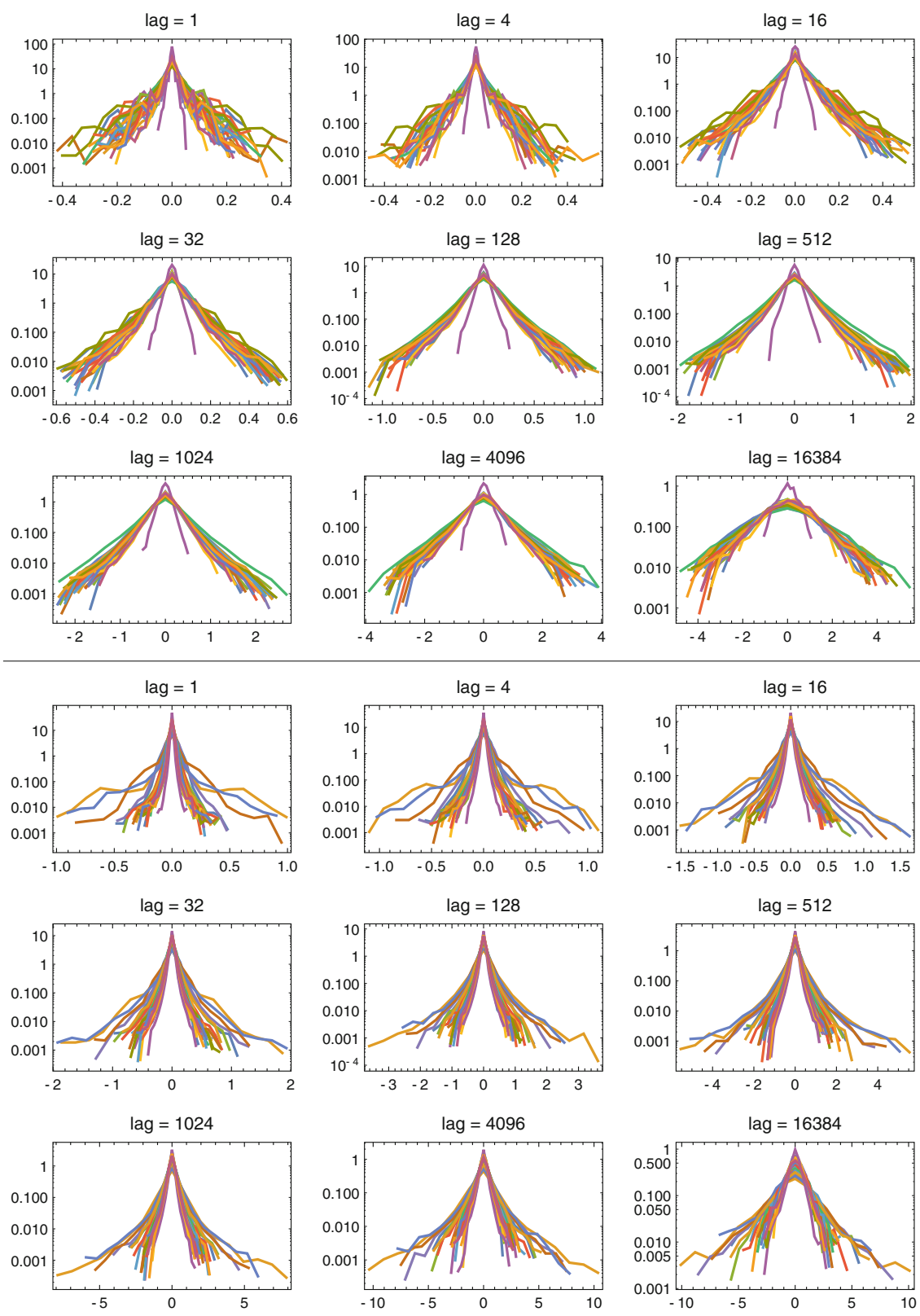

Fig. 6 Probability densities on a log-scale for the log-returns of all 29 assets at various lags ranging from 1 to $16384 \mathrm{~s}$. The top and bottom halfs represent the "pre" and "post" datasets, respectively. The log-returns have been multiplied with 100 in order to un-clutter the labeling of the $x$-axes 

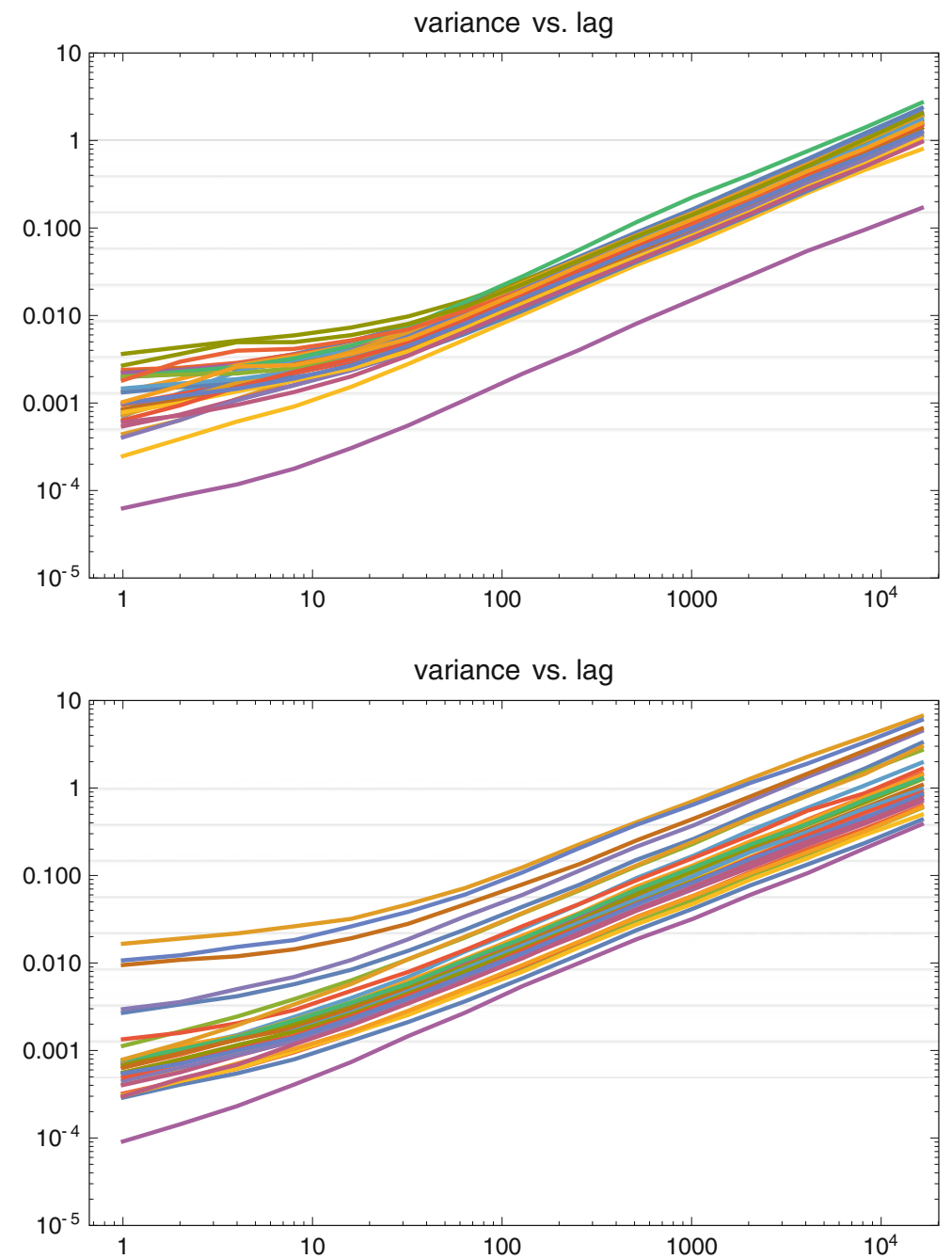

Fig. 7 The variance of the log-returns for the 29 assets as a function of the lag displayed in a double logarithmic representation. The top and bottom graphs represent the "pre" and "post" datasets, respectively

\subsection{Modelling Turbulent Velocity Time Series}

A specific time-wise version of (1), called Brownian semistationary processes has been proposed in $[18,19]$ as a model for turbulent velocity time series. It was shown that BSS processes in combination with continuous cascade models (exponentials of certain trawl processes) are able to qualitatively capture some main stylized features of turbulent time series. 

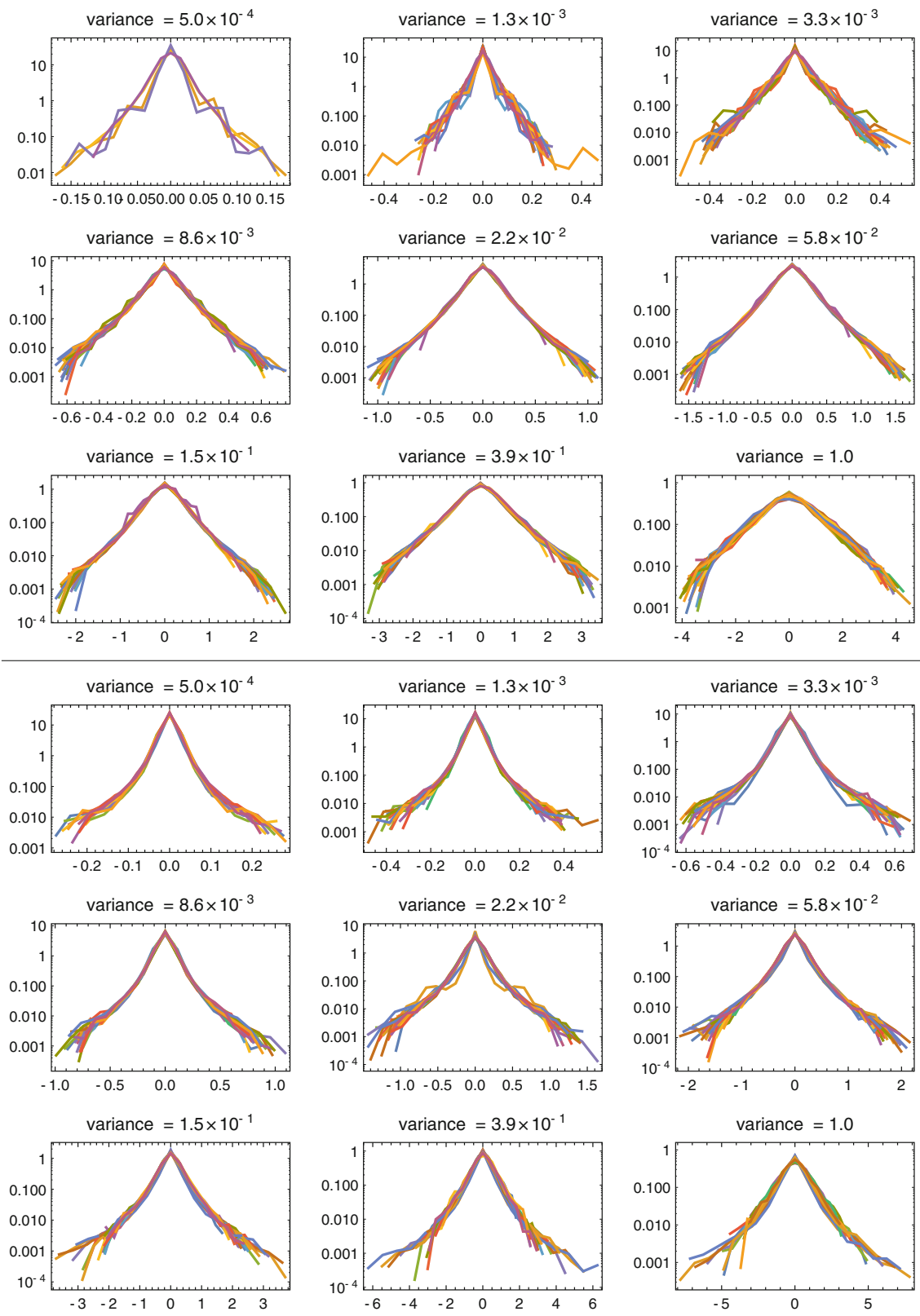

Fig. 8 Probability densities on a log-scale of log-returns where the lag for each asset has been chosen such that the variances of the assets in each subplot is the same. The chosen variances are also displayed in Fig. 7 as horizonal lines. For the smallest and largest variances, not all dataset are present since for some datasets those variances are not attained. Top "pre", bottom "post" 
Recently this analysis has been extended to a quantitative comparison with turbulent data [31, 35]. More specifically, based on the results for the energy dissipation oulined in Sect.4.1, BSS processes have been analyzed and compared in detail to turbulent velocity time series in [35] by directly estimating the model parameters from data. Here we briefly summarize this analysis.

Time series of the main component $v_{t}$ of the turbulent velocity field are modelled as a BSS process of the specific form

$$
v(t)=v(t ; g, \sigma, \beta)=\int_{-\infty}^{t} g(t-s) \sigma(s) B(d s)+\beta \int_{-\infty}^{t} g(t-s) \sigma(s)^{2} d s=: R(t)+\beta S(t)
$$

where $g$ is a non-negative $L^{2}\left(\mathbb{R}_{+}\right)$function, $\sigma$ is a stationary process independent of $B, \beta$ is a constant and $B$ denotes standard Brownian motion. An argument based on quadratic variation shows that when $g(0+) \neq 0$, then $\sigma^{2}$ can be identified with the surrogate energy dissipation, $\sigma^{2}=\varepsilon$, where $\varepsilon$ is the process given by (17). The kernel $g$ is specified as a slightly shifted convolution of gamma kernels [30],

$$
\begin{aligned}
g(t) & =g_{0}\left(t+t_{0}\right), \\
g_{0}(t) & =a t^{v_{1}+v_{2}-1} \exp \left(-\lambda_{2} t\right){ }_{1} F_{1}\left(v_{1}, v_{1}+v_{2},\left(\lambda_{2}-\lambda_{1}\right) t\right) 1_{(0, \infty)}(t)
\end{aligned}
$$

with $a>0, v_{i}>0$ and $\lambda_{i}>0$. Here ${ }_{1} F_{1}$ denotes the Kummer confluent hypergeometric function. The shift is needed to ensure that $g(0+) \neq 0$.

The data set analysed consists of one-point time records of the longitudinal (along the mean flow) velocity component in a gaseous helium jet flow with a Taylor Reynolds number $R_{\lambda}=985$. The same data set is also analyzed in [31] and the estimated parameters there are used to specify $\sigma^{2}=\varepsilon$ in (28). The remaining parameters for the kernel $g$ and the constant $\beta$ can then be estimated from the second and third order structure function, that is, the second and third order moments of velocity increments. In [35] it is shown that the second order structure function is excellently reproduced and that the details of the third order structure function are well captured. It is important to note that the model is completely specified from the energy dissipation statistics and the second and third order structure functions.

The estimated model for the velocity is then succesfully compared with other derived quantities, including higher order structure functions, the distributions of velocity increments and their evolution as a function of lag, the so-called Kolmogorov variable and the energy dissipation, as prediced by the model.

\section{Conclusion and Outlook}

The present paper highlights some of the most recent developments in the theory and applications of Ambit Stochastics. In particular, we have discussed the existence of the ambit fields driven by metatime changed Lévy bases, selfdecomposability of random fields [13], applications of BSS processes in the modelling of turbulent time 
series [35] and new results on the distributional collapse in financial data. Some of the topics not mentioned here but also under development are the integration theory with respect to time-changed volatility modulated Lévy bases [7]; integration with respect to volatility Gaussian processes in the White Noise Analysis setting in the spirit of [34] and extending [6]; modelling of multidimensional turbulence based on ambit fields; and in-depth study of parsimony and universality in BSS and LSS processes motivated by some of the discussions in the present paper.

Open Access This chapter is distributed under the terms of the Creative Commons Attribution Noncommercial License, which permits any noncommercial use, distribution, and reproduction in any medium, provided the original author(s) and source are credited.

\section{References}

1. Barndorff-Nielsen, O.E.: Processes of normal inverse Gaussian type. Finance Stoch. 2(1), 4168 (1998)

2. Barndorff-Nielsen, O.E.: Stationary infinitely divisible processes. Braz. J. Probab. Stat. 25(3), 294-322 (2011)

3. Barndorff-Nielsen, O.E.: Notes on the gamma kernel. Research Report 3, Thiele Centre for Applied Mathematics in Natural Science. (Aarhus University, 2012)

4. Barndorff-Nielsen, O.E., Blæsild, P., Schmiegel, J.: A parsimonious and universal description of turbulent velocity increments. Eur. Phys. J. B 41, 345-363 (2004)

5. Barndorff-Nielsen, O.E., Lunde, A., Shephard, N., Veraart, A.E.D.: Integer-valued trawl processes: a class of infinitely divisible processes. Scand. J. Stat. 41(3), 693-724 (2013)

6. Barndorff-Nielsen, O.E., Benth, F.E., Szozda, B.: Stochastic integration for volatility modulated Brownian-driven processes via white noise analysis. Infin. Dimens. Anal. Quantum Probab. 17(2), 1450011 (2014)

7. Barndorff-Nielsen, O.E., Benth, F.E., Szozda, B.: Integration with respect to time-changed volatility modulated Lévy-driven Volterra processes. (In preparation, 2015)

8. Barndorff-Nielsen, O.E., Benth, F.E., Veraart, A.E.D.: Recent advances in ambit stochastics with a view towards tempo-spatial stochastic volatility/intermittency. Banach Center Publications (To appear). (2015)

9. Barndorff-Nielsen, O.E., Maejima, M., Sato, K.: Infinite divisibility for stochastic processes and time change. J. Theor. Probab. 19, 411-446 (2006)

10. Barndorff-Nielsen, O.E., Pakkanen, M., Schmiegel, J.: Assessing relative volatility/intermittency/energy dissipation. Electron. J. Stat. 8, 1996-2021 (2014)

11. Barndorff-Nielsen, O.E., Pedersen, J.: Meta-times and extended subordination. Theory Probab. Appl. 56(2), 319-327 (2010)

12. Barndorff-Nielsen, O., Pérez-Abreu, V., Thorbjørnsen, S.: Lévy mixing. Lat. Am. J. Probab. Math. Stat. 10, 921-970 (2013)

13. Barndorff-Nielsen, O.E., Sauri, O., Szozda, B.: Selfdecomposable fields. (In preparation, 2015)

14. Barndorff-Nielsen, O.E., Shephard, N.: Non-Gaussian Ornstein-Uhlenbeck-based models and some of their uses in financial economics. J. Royal Stat. Soc. 63, 167-241 (2001)

15. Barndorff-Nielsen, O.E., Shephard, N., Sauri, O., Hedevang, E.: Levy-driven Models with volatility. (In preparation, 2015)

16. Barndorff-Nielsen, O.E., Schmiegel, J.: Time change and universality in turbulence. In: Research Report 15, Thiele Centre for Applied Mathematics in Natural Science, Aarhus University. (2006) 
17. Barndorff-Nielsen, O.E., Schmiegel, J.: Ambit processes; with applications to turbulence and tumour growth. In: Benth, F.E., Nunno, G.D., Linstrøm, T., Øksendal, B., Zhang, T. (eds.) Stochastic Analysis and Applications: The Abel Symposium 2005, pp. 93-124. Springer, Heidelberg (2007)

18. Barndorff-Nielsen, O.E., Schmiegel, J.: A stochastic differential equation framework for the timewise dynamics of turbulent velocities. Theory Probab. Its Appl. 52, 372-388 (2008)

19. Barndorff-Nielsen, O.E., Schmiegel, J.: Brownian semistationary processes and volatility/intermittency. In: Albrecher, H., Rungaldier, W., Schachermeyer, W. (eds.) Advanced financial modelling. Radon Series on Computational and Applied Mathematics, vol. 8. pp. 1-26. W. de Gruyter, Berlin (2009)

20. Barndorff-Nielsen, O.E., Hedevang, E., Schmiegel, J.: Incremental similarity and turbulence. (Submitted.) (2015)

21. Barndorff-Nielsen, O.E., Schmiegel, J., Shephard, N.: Time change and Universality in turbulence and finance. In: Research Report 18, Thiele Centre for Applied Mathematics in Natural Science, Aarhus University. (2006)

22. Barndorff-Nielsen, O.E., Shiryaev, A.N.: Change of Time and Change of Measure. World Scientific, Singapore (2010)

23. Basse-O'Connor, A., Graversen, S.E., Pedersen, J.: Stochastic integration on the real line. Theory Probab. Its Appl. 58, 355-380 (2013)

24. Basse-O'Connor, A., Lachièze-Rey, R., Podolskij, M.: Limit theorems for stationary increments Lévy driven moving average processes. (To appear). (2015)

25. Chong, C., Klüppelberg, C.: Integrability conditions for space-time stochastic integrals: theory and applications. Bernoulli. In press (2015)

26. Corcuera, J.M., Hedevang, E., Pakkanen, M., Podolskij, M.: Asymptotic theory for Brownian semi-stationary processes with application to turbulence. Stochast. Proc. Their Appl. 123, 2552-2574 (2013)

27. Dhruva, B.R.: An experimental study of high Reynolds number turbulence in the atmosphere. $\mathrm{PhD}$ Thesis, Yale University (2000)

28. Eberlein, E.: Application of generalized hyperbolic Lévy motions to finance. In: BarndorffNielsen, O.E. et al. (eds.) Lévy Processes. Theory and Applications, 319-336. Birkhäuser (2001)

29. Hansen, P.R., Lunde, A.: Realized variance and market microstructure noise. J. Bus. Econ. Stat. 24(2), 127-161 (2006)

30. Hedevang, E.: Stochastic modelling of turbulence: with applications to wind energy. Ph.D thesis. Aarhus University (2012)

31. Hedevang, E., Schmiegel, J.: A causal continuous-time stochastic model for the turbulent energy cascade in a helium jet flow. J. Turbul. 14(11), 1-26 (2014)

32. Jurek, Z.J.: The random integral representation conjecture a quarter of a century later. Lith. Math. J. 51, 362-369 (2011)

33. von Karman, T.: Progress in the statistical theory of turbulence. J. Marine Res. 7, 252-264 (1948)

34. Lebovits, J.: Stochastic calculus with respect to gaussian processes (2014). arXiv:1408.1020

35. Márquez, J.U., Schmiegel, J.: Modelling turbulent time series by BSS-processes. (Submitted.) (2015)

36. Podolskij, M.: Ambit fields: survey and new challenges. (To Appear in XI Proceedings of Symposium of Probability and Stochastic Processes) (2014)

37. Rajput, B.S., Rosiński, J.: Spectral representations of infinitely divisible processes. Probab. Theory Rel. Fields 82, 451-487 (1989)

38. Sato, K., Yamazato, M.: Operator selfdecomposable distributions as limits distributions of processes of Ornstein-Uhlenbeck type. Stoch. Process. Appl. 17(1), 73-100 (1984)

39. Steutel, F.W., van Harn, K.: Infinite Divisibility of Probability Distributions on the Real Line. Marcel Dekker (2004)

40. Veraart, A.E.D., Veraart, L.A.M.: Modelling electricity day-ahead prices by multivariate Lévy semistationary processes. In: Benth, F.E., Kholodnyi, V., Laurence, P. (eds.) Quantitative Energy Finance, pp. 157-188. Springer (2014) 